\title{
CLINICAL REFLECTION \\ Communication skills training for healthcare students working with people with intellectual disabilities
}

\author{
Elizabeth Metcalf \& Robert Colgate
}

Elizabeth Metcalf, MBBCh, MRCGP, MSc, DRCOG, DFSRH, FAcadMEd, is Clinical Assessments Lead at the School of Medicine, Cardiff University, and a general practitioner. She represents Cardiff University on the UK Council of Clinical Communication, UK Counci of Clinical Skills Teachers and the Medical Schools Council Assessment Alliance. Research interests include clinical communication skills and authentic clinical assessments. Robert Colgate, MBBCh, FRCPsych, MSc, PGCE, is a consultant in old age liaison psychiatry employed by Abertawe Bro Morgannwg (ABM) University Health Board in Bridgend, Wales. He is also Associate Medical Director for Mental Health with the Welsh Health Specialised Services

Committee (WHSSC).

Correspondence Dr Elizabeth

Metcalf, Cardiff University School of Medicine, Centre for Medical

Education, College of Biomedical and Life Sciences, Cochrane Building, Heath Park, Cardiff CF14 4YS, UK. Email: metcalfep@cf.ac.uk

\section{Copyright and usage} (c) Cardiff University Research and Innovation Services 2018

\begin{abstract}
SUMMARY
Effective communication skills are essential for all healthcare professionals. Specialist teaching on communicating with people with intellectual disabilities is often lacking, resulting in poorer healthcare and worse outcomes than in the general population. Working with professionally trained actors with intellectual disabilities, we developed an interprofessional workshop that sought to provide authentic communication skills training to enable healthcare students from various disciplines to communicate effectively with patients of all abilities. In a survey, students reported that they found the workshops rewarding and confidence-building, and that they learnt more about the roles of their interprofessional colleagues.
\end{abstract}

\section{DECLARATION OF INTEREST}

None.

\section{KEYWORDS}

Education and training; intellectual disability; primary care.

Doctors must 'communicate clearly, sensitively and effectively with all patients' (General Medical Council 2018). Specific skills are required when communicating with vulnerable groups, for example patients with intellectual disabilities (also known as learning disabilities in UK health services). These skills are crucial and have a direct impact on healthcare outcomes (Watkins 2016). People with intellectual disabilities frequently have multiple healthcare needs (Disability Rights Commission 2006), many of which reduce life expectancy. These individuals receive advice and care from health and social care professionals from multiple disciplines. Mencap (2007) explored the deaths of six people with intellectual disabilities and identified factors that contributed to their premature or preventable deaths. The report highlighted healthcare staff's lack of awareness of patient's needs, healthcare inequalities and a failure to comply with the Disability Discrimination Act 1995 (which has since been replaced by the Equality Act 2010). At the end of its report, Mencap made a number of recommendations - including provision of competency-based training about intellectual disabilities for all healthcare professionals, with the involvement of patients, their carers and families in this training. Despite these recommendations, the Learning Disability Mortality Review (LeDeR) report (2017) (University of Bristol 2016-2017) highlighted that these problems remain a barrier to healthcare for people with intellectual disabilities.

The opportunity for medical students on clinical placement to meet and communicate with patients with diverse intellectual disabilities is relatively limited, particularly early in the undergraduate medical course. We therefore developed a pioneering partnership between Cardiff University School of Medicine, Cardiff Metropolitan University, Cardiff School of Sport \& Health Sciences and Hijinx, a Welsh theatre company that includes professionally trained actors with intellectual disabilities, that resulted in a workshop offering a new interprofessional learning experience.

The key principle of our approach to 'skills-based' (Silverman 2013) communication skills training in Cardiff is to help students to build constructive, therapeutic relationships with their patients and to learn how to gather and share information with patients. We seek to challenge unconscious beliefs and, where necessary, to change students' attitudes. Students learn how various medical conditions can affect a patient's ability to communicate and that developing their own communication techniques can improve clinical outcomes. From the outset, we aim for our students to be 'accurate, efficient and compassionate'.

\section{The communication skills workshop}

A pilot workshop delivered to third-year medical students in 2015 received strong positive feedback from participants. Evidence from the literature promotes the introduction of interprofessional communication skills training - in which healthcare professionals from different disciplines learn with, 
Information given to actor: You have come along to the Accident and Emergency department at your local hospital. Earlier today, a strong gust of wind blew open a door that struck you hard on the left side of your head just in front of your ear. You did feel a bit dizzy and sick at first but now feel a lot better. You were not knocked out. You have not been sick and your headache has almost gone.

Fortunately there is only a nasty bruise and no blood!

The students will complete three tasks.

1. They will ask about what has happened and give you some simple advice about your head injury.

2. They will ask to check your pupil reactions (by shining a torch light in your eyes).

3. They will explain about warning signs after a head injury.

When you speak to the students, just be yourself! Listen carefully to what they say. If you have any questions, make sure that you ask them to explain and if you do not understand what they are doing, make sure that they explain again!

At the end, tell the students what you think they explained best about what has happened to you. If there is time, ask the students about what to do tonight: should you ask someone to wake you up during the night to check you are alright?

Information given to student 1: You have been asked to see an adult who has been struck hard on the side of the head by a door. Introduce yourself to this patient and confirm the sequence of events earlier today. Ask about headache, nausea or vomiting and unfamiliar weakness affecting an arm or a leg. Explain that one further examination is needed (you will want to check pupil reactions) before this patient can safely return home.

Information given to student 2: Next confirm that the pupil reactions are normal. Explain what you are going to do and ensure that you gain informed consent. Discuss a summary of your findings with the patient.

Information given to student 3: You have now confirmed that there are no warning signs at present and it is safe for this patient to return home. Provide a simple list of the warning signs which might suggest a more severe head injury - increasing or unexpected sleepiness, persistent or worsening headache, vomiting more than once, fits, faints or blackouts or weakness of any limb.

Extension task: explore the capacity of this patient to consent to prescription of simple analgesia for headache. Answer any further questions they may have.

A scenario given to an actor in a communication skills workshop.

from and about each other to improve collaboration and quality of care - in an academic setting (Keller 2013), particularly when it focuses on the development of innovative, experiential learning opportunities and those that incorporate standardised patients and feedback from faculty facilitators (Solomon 2011). We therefore felt that introducing an interprofessional element would enrich the opportunities for students and improve awareness of the expertise offered by colleagues in other disciplines. As a result, in November 2016 the new interprofessional session was introduced to 300 medical students during their chronic disease teaching block and to approximately 40 SALT students per year.

Mixed groups of fourth-year medical and thirdyear SALT students undertake a 90 min communication skills workshop, during which they have the opportunity to role-play with actors with developmental intellectual disabilities and receive feedback from tutors, actors and peers. Students are encouraged to communicate with patients using recognised effective strategies, including the involvement of other disciplines, for example speech and language therapists and other allied health professionals, which nurtures their skills and confidence in providing healthcare to vulnerable patients while maintaining the patients' dignity.

Hijinx actors rehearse relevant scenarios and receive training ahead of the teaching sessions (Fig. 1). The emphasis of our teaching is to encourage the development of communication skills and the importance of using simple comprehensible language, repeated where necessary. Students learn and practise a method of actively confirming the patient's retention and understanding that is considered good practice and often used by surgeons. For this technique, the medical practitioner asks the patient to explain what they have understood about the procedure or diagnosis to an accompanying relative/carer or, if none is present, as a hypothetical exercise. This allows the practitioner to assess whether the patient has indeed understood what was explained to them and to address any areas where understanding has been demonstrated to still be lacking (Royal College of Surgeons 2015). Students are encouraged to consider what the barriers to effective communication may be 


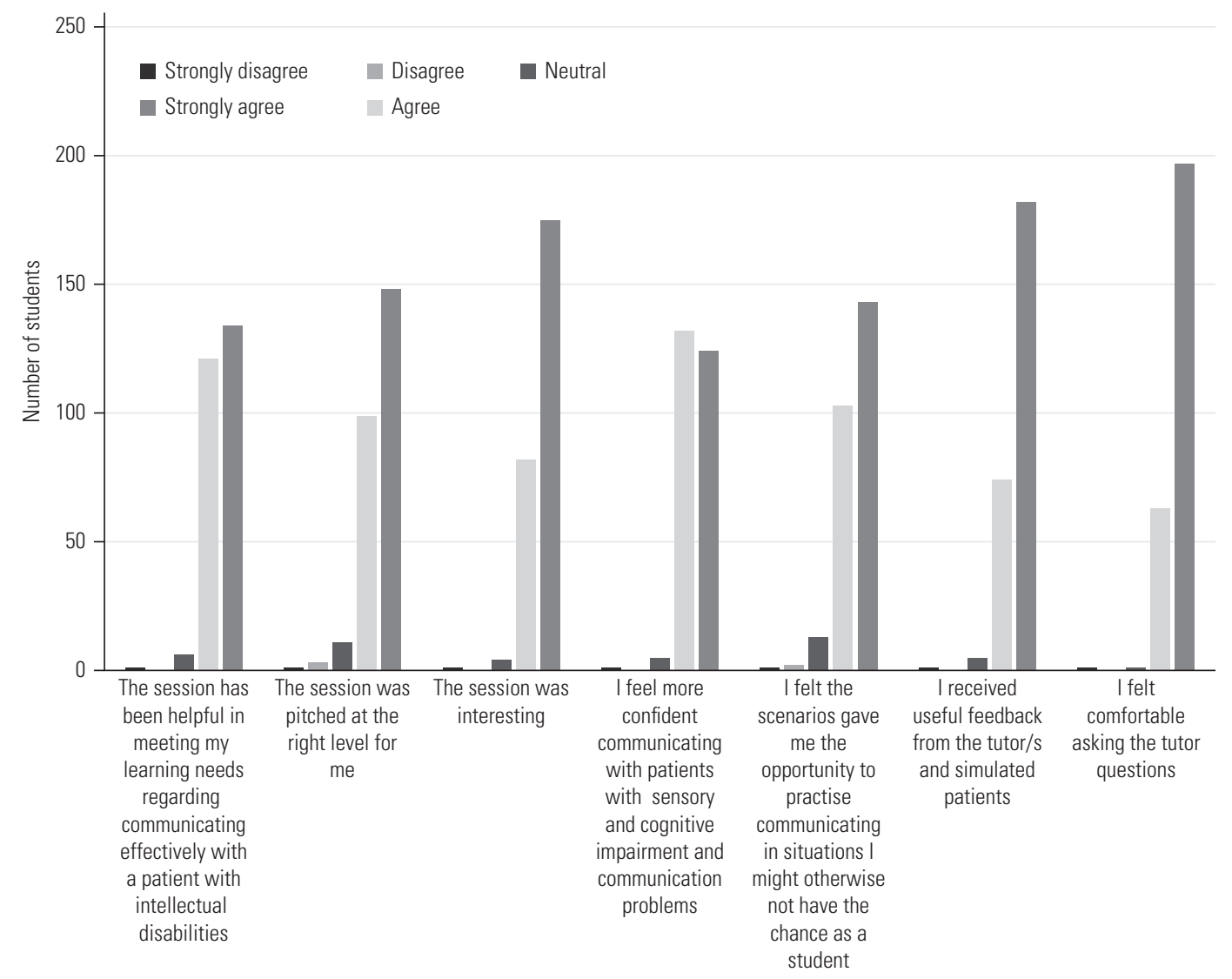

FIG 2 Students' $(n=262)$ evaluations of the interprofessional workshop on communication skills delivered to medical students and speech and language therapy students in Cardiff.

and identify effective methods to engage rapport quickly and to maintain it. The educational priority is consolidation of existing communication skills and development of knowledge.

\section{Evaluation of the workshops}

A significant barrier to accessing healthcare faced by people with intellectual disabilities arises from the language that healthcare professionals use and how much time they give individuals to communicate with them. Communication skills training allows students to develop ways of adapting language and highlights the importance of supplementing spoken communication, for example with visual aids including diagrams and with effective nonverbal communication. Role-play allows them to experience communication difficulties for themselves and provides an opportunity to practise how to facilitate good communication. An unpublished survey of students who had participated in our training workshops showed that they found this teaching rewarding and that the interprofessional nature of the course enabled them to learn more about the roles of their colleagues in other disciplines (Fig. 2). It also revealed a significant increase in the confidence and a competence change measured in terms of an increase informally observed by tutors of students in their interactions with patients with intellectual disabilities. Students find the teaching enjoyable and valuable, while the collaboration with Hijinx provides genuine and sustained employment for actors with intellectual disabilities, improving their financial independence, self-esteem and well-being.

\section{Take-home message}

Our communication skills workshops provide students with a rare opportunity to practise and improve their communication skills to meet the healthcare needs of people with intellectual disabilities. They also enable Hijinx actors to make a valuable, lasting contribution to society, something rarely offered to adults with intellectual disabilities.

\section{References}

Disability Rights Commission (2006) Equal Treatment: Closing the Gap A Formal Investigation into Physical Health Inequalities Experienced by People with Learning Disabilities and/or Mental Health Problems. Disability Rights Commission.

General Medical Council (2018) Outcomes for Graduates 2018. GMC.

Keller KB, Eggenberger TL, Belkowitz J, et al (2013) Implementing successful interprofessional communication opportunities in health care 
education: a qualitative analysis International Journal of Medical Education, 4: 253-9

Mencap (2007) Death by Indifference: Following Up the Treat Me Right! Report. Mencap.

Royal College of Surgeons (2015) Consent: Supported Decision-Making. A Guide to Good Practice. Royal College of Surgeons.

Silverman J, Kurtz S, Draper J (2013) Skills for Communicating with Patients (3rd edn). CRC Press
Solomon P Salfi J (2011) Evaluation of an interprofessional education communication skills initiative. Education for Health, 24: 616

University of Bristol (2017) The Learning Disabilities Mortality Review (LeDeR) Programme: Annual Report 2016-2017.

Watkins LW, Colgate R (2016) Improving healthcare for people with intellectual disabilities: the development of an evidence-based teaching programme. Advances in Mental Health and Intellectual Disabilities, 10: $333-41$. 